\title{
Nature and Development of Econophysics: A Short Report
}

\author{
Paolo Di Sia \\ Faculty of Economics and Management, Free University of Bozen-Bolzano, Bozen-Bolzano, Italy \\ Email: paolo.disia@yahoo.it
}

Received January 4, 2012; revised February 2, 2012; accepted February 14, 2012

\begin{abstract}
In this work I offer a brief non-technical, but rigorous report of econophysics; starting from the relations between physics and economics, I focus my considerations about the studied problems and the utilized methods, arriving to present an interesting view concerning the present of the discipline and some lines of the current research referred to theoretical physics.
\end{abstract}

Keywords: Econophysics; Economics; Theoretical Physics; Education

\section{Introduction}

The econophysics is a rather complex discipline and of not simple definition, even if however have been done various attempts to define it. As "inventors" of the term, Rosary Mantegna and H. Eugene Stanley have defined the econophysics as "a neologism that denotes the activeties of physicists who are working on economics problems to test a variety of new conceptual approaches deriving from the physical sciences" [1]. The more usual way for defining the econophysics is as a "multidisciplinary (or "interdisciplinary") discipline”; such definition has a sociological orientation and it resembles for some aspects to the contrast among socio-economics and social economics (the first broadly treated by the sociologists, the second bye the economists).

The econophysics involves physicists dealing with economics through theories of physics domain, raising the question on how the two disciplines are related. From a theoretical point of view, it has traditionally considered that the two disciplines belong to the two distinct categories of physical (or natural, or experienced) sciences and social sciences. Such distinction seems however to be not entirely appropriate, being physics areas (as the speculative theoretical physics) which are not experimentally testable (only in a partial and indirect way) and on the other hand the growing use of experiments in economics, although not scientifically controlled as in the domain of physics, chemistry and biology.

Another point of view on relationability of the two disciplines regards which parts of the universe they study within the great hierarchy of the structure of reality. Going from the microscopic to the macroscopic perspective, the physics studies the two most great extremes: from the infinitesimal level of subparticle-subatomic physics to the infinity tending level of the cosmic extragalactic astrophysics, with theories such as string theory and quantum gravity, which seek to treat both levels simultaneously.

Starting from the microscopic level of physics it passes through chemistry, molecular and organic biology, for arriving to psychology, economics, political science, sociology, ecology, climatology and geology, over the scale of earth planet. In this hierarchy we find the social sciences at an intermediate scale, with the economics in a crucial position between the individual behavior of the person and the population as a whole.

From the point of view of an intellectual hierarchy of the disciplines, starting from the most abstract and theoretical for arriving to the most "practical", we start from pure mathematics and speculative theoretical physics passing through the applied disciplines (including statistics and computer science), for arriving at applied physics and chemistry, then economics, which is perhaps, between social sciences, the most mathematical oriented. This implicit "intellectual superiority" of physics with respect to economics could open the door to potential conflicts between the two disciplines; on a hand, the econophysicists argue that economists are not disposal to accept and/or deal data or facts not in line with predicttions of their theories (sometimes outdated theories), on the other hand, economists who study the same data and models of econophysicists believe that the physicists are not always aware of their work and of the true nature of economic theories. The future econophysics development must include also the overcoming of these prejudices through communication, research and mutual efforts.

\section{Studied Problems and Utilized Methods}

The econophysicists work on economic problems with 
new conceptual approaches deriving from the physical sciences. The more extensively studied thematics were so far the distributions of investments in financial markets [2-4], the distribution of income and wealth [5,6], the distribution of economic falls, the changes in economic growth rate [7], the urban size distribution [8] and the distribution of scientific knowledge [9]. Models and theories mainly used have been the statistical mechanics [10], the models for the study of earthquakes and selforganized critical models of dynamics [11].

One of the clearest differences between the two approaches (econophysical and economic approaches) has focused on the conviction of the first one that many of the above mentioned phenomena can be best described using scaling laws involving non-gaussian distributions, which exhibit asymmetry and leptokurtosis rather than gaussian distributions; this situation covers also the area of financial investment distributions. The "mean and variance" approach is applied to the risk analysis and leads to conceptual results as the Black-Scholes formula [12], extensively used in the "option pricing" models and in the financial markets sector.

\section{On Temporal Precedence between Physics and Economics}

Another considered problem regards the temporal precedence for these disciplinary developments; historically it results that a physical model in some cases has arrived from the economics, and some standard economics models arrive from physics. Therefore "econophysics" might be simply a new name for something that is actually using from long time.

Many examples can be taken at this regard: Pareto was an economist and sociologist, but he studied previously to many physicists the distributions exhibiting "scaling" laws; Bachelier was a mathematician who studied financial markets, but he started to develop the brownian motion idea parallely to the physicists. On the other hand, Osborne [13] was a physicist who played an important role in strengthening the standard financial economics, which uses the gaussian brownian motion model; the founder of general equilibrium theory in economics, Léon Walras [14], has been deeply influenced by the physicist Luigi Poinsot [15] in the formulation of this central concept.

The father of the american mathematical economics (in its neoclassical form), Irving Fisher [16], was a student of one of the fathers of statistical mechanics, J. Willard Gibbs [17]. The peak of this transfer of physical concepts in standard neoclassical economics came with the publication of "Paul Samuelson's Foundations of Economic Analysis" in 1947 [18], Samuelson having been originally an undergraduate physics student at the
University of Chicago. Also the subsequent more direct introduction of statistical mechanics applications in economics occurred by the same economists [19].

Econophysics is an evolving discipline and it will continue an important development whatever is its long-term evolution. It was raised a particular issue concerning its relationship with the economics profession: in particular, it is something that is acceptable for the orthodox economic theory, or is it something heterodox? This question requires some terminological definitions which involve two aspects in the used definitions for the understanding of these terms: the intellectual and sociological aspects. Some authors [20] state that "orthodox" refers to a purely intellectual category. The "orthodox economic theory" is a stable and more or less internally consistent ideas set, which was widely accepted, at least in the recent past.

A clear way for identify these issues is to see how they are presented in the more used manuals (at university level and not); it seems therefore that the answer to the question is "yes". Econophysics certainly contests the old established orthodoxy, and the "staticity" found sometimes in conventional economics textbooks. In every case the issue affects on econophysics in a more "philosophical" than practical way, therefore a lot of important economists have taken seriously the actually econophysics research. Many economists are aware of the fact that the physical theories are able to explain phenomena unexplainable from the economical theories.

\section{The Present and the Future of Econophysics}

It is not possible to predict with precision about the possibility that ideas specifically developed by economists will influence the physics in the future, although if this cannot be excluded "a priori". However, the "articulated" way of the interaction between economics and physics, occurred in past, seems to attenuate passing the time, also through the development of the discipline. This interacttion reflects for many aspects the more extensive reality of the mutual influence of various disciplines through the complexity theory and other transdisciplinary theories, such as the non-linear dynamics, the game theory and other mathematically more sophisticated approaches [21].

It is so to be expected that the econophysics will continue to grow and increase in influence in the future; for this to happen, economists will need to know ideas and physical theories and accept them, and physicists will learn more of what has been done by economists.

The relationships between game theory and quantum mechanics proposed quantized mathematical tools with which it seems possible to describe and understand not 
only quantum physical systems, but also to explain more correctly biological and economical processes, including theories like game and evolutionary dynamics. So it becomes possible to utilize some concepts and definitions of quantum mechanics and modern physics for the best understanding of the behaviour of economics. In this way it is maybe possible to understand nature like a game in which the players compete for a common welfare and are searching for an equilibrium [22].

The last research is bringing to a new relativistic direction in quantum econophysics, in which it is offered an important change of the classical paradigms in mathematical modeling of socio-economic systems. In the classical physics it holds the hypothesis that the immediate values of all physical quantities, characterizing system's state, exist and can be accurately measured in principle. Non-relativistic quantum mechanics does not reject the existence of the immediate values of the classical physical quantities, but add via the uncertainty principle the impossibility of a simultaneous measure of them. Relativistic quantum mechanics rejects the existence of the immediate values of any physical quantity in princeple, and as consequence the notion of the system state. Scientists have started in these years an econophysical analysis of the conceptual fundamentals and mathematiccal apparatus of the classical and modern physics, subjected to socio-economic and regarding socio-economic modeling problems, showing that notions like that of the physical quantity operator become actually the primary fundamental conception (the operator is a mathematical image of the procedure, i.e. the action), the description of the system dynamics becomes discrete, the prediction of the future becomes impossible, setting aside the aftereffect, i.e. the memory. All these scientific developments are suggesting new paradigms of the economical-mathematical modeling [23].

\section{REFERENCES}

[1] R. N. Mantegna and H. E. Stanley, "An Introduction to Econophysics: Correlations and Complexity in Finance,” Cambridge University Press, Cambridge, 2000.

[2] P. Mirowski, "More Heat than Light: Economics as Social Physics," Cambridge University Press, Cambridge, 1989. doi:10.1017/CBO9780511559990

[3] J.-P. Bouchaud and R. Cont, "A Langevin Approach to Stock Market Fluctuations and Crashes," European Physical Journal B, Vol. 6, No. 4, 2002, pp. 543-550. doi:10.1007/s100510050582

[4] D. Sornette, "Why Stock Markets Crash: Critical Events in Complex Financial Systems," Princeton University Press, Princeton, 2003.

[5] M. Levy and S. Solomon, "New Evidence for the Power-Law Distribution of Wealth,” Physica A, Vol. 242,
No. 1, 1997, pp. 90-94 doi:10.1016/S0378-4371(97)00217-3

[6] A. Chatterjee, S. Yarlagadda and B. K. Chakrabarti, Eds., "Econophysics of Wealth Distributions," Springer, Milan, 2005.

[7] D. Canning, L. A. N. Amaral, Y. Lee, M. Meyer and H. E. Stanley, "Scaling the Volatility of GDP Growth Rates," Economics Letters, Vol. 60, No. 3, 1998, pp. 335-341. doi:10.1016/S0165-1765(98)00121-9

[8] X. Gabaix, “Zipf's Law for Cities: An Explanation,” Quarterly Journal of Economics, Vol. 114, No. 3, 1999, pp. 739-767. doi:10.1162/003355399556133

[9] V. Plerou, L. A. N. Amaral, P. Gopakrishnan, M. Meyer and H. E. Stanley, "Similarities between the Growth Dynamics of University Research and of Competitive Economic Activities,” Nature, Vol. 400, 1999, pp. 433-437. doi:10.1038/22719

[10] F. Spitzer, "Random Fields and Interacting Particle Systems,” American Mathematical Society, Providence, 1971.

[11] P. Bak, "How Nature Works: The Science of Self-Organized Criticality,” Copernicus Press for Springer-Verlag, New York, 1996.

[12] F. Black and M. Scholes, "The Pricing of Options and Corporate Liabilities,” Journal of Political Economy, Vol. 81, No. 3, 1973, pp. 637-654. doi:10.1086/260062

[13] M. F. M. Osborne, "Brownian Motion in the Stock Market,” Operations Research, Vol. 7, No. 2, 1959, pp. 145173. doi:10.1287/opre.7.2.145

[14] L. Walras, “Éléments d’Économie Politique Pure,” F. Rouge, Lausanne, 1874.

[15] L. Poinsot, “Éléments de Statique,” 8th Edition, Bachelier, Paris, 1842.

[16] I. Fisher, “The Theory of Interest,” Augustus M. Kelly, New York, 1930.

[17] J. W. Gibbs, "Elementary Principles of Statistical Mechanics,” Yale University Press, New Haven, 1902.

[18] P. A. Samuelson, "Foundations of Economic Analysis," Harvard University Press, Cambridge, 1947.

[19] H. Föllmer, "Random Economies with Many Interacting Agents,” Journal of Mathematical Economics, Vol. 1, No. 1, 1974, pp. 51-62. doi:10.1016/0304-4068(74)90035-4

[20] D. Colander, R. P. F. Holt and J. B. Rosser Jr., “The Changing Face of Economics: Conversations with Cutting Edge Economists,” University of Michigan Press, Ann Arbor, 2004.

[21] S. A. Kauffman, "The Origins of Order: Self-Organization and Selection in Evolution," Oxford University Press, Oxford, 1993.

[22] H. E. Stanley, "Exotic Statistical Physics: Applications to Biology, Medicine, and Economics,” Physica A, Vol. 285, 2000, pp. 1-17. doi:10.1016/S0378-4371(00)00341-1

[23] V. P. Maslov, "Econophysics. Modern Physics in Search of Economic Theory,” MIFI, Moscow, 2007. 